\title{
ON GEOMETRIC GRAPH RAMSEY NUMBERS
}

GYULA KÁROLYI ${ }^{1}$ Department of Algebra and Number Theory, Eötvös University, Pázmány P. sétány 1/C, Budapest, H-1117 Hungary

VERA ROSTA Alfréd Rényi Institute of Mathematics, Reáltanoda utca 13-15, Budapest, H-1053 Hungary

Abstract. For any two-colouring of the segments determined by $3 n-3$ points in general position in the plane, either the first colour class contains a triangle, or there is a noncrossing cycle of length $n$ in the second colour class, and this result is tight. We also give a series of more general estimates on off-diagonal geometric graph Ramsey numbers in the same spirit. Finally we investigate the existence of large noncrossing monochromatic matchings in multicoloured geometric graphs.

\section{INTRODUCTION}

For any finite sequence $G_{1}, G_{2}, \ldots, G_{t}$ of simple graphs, $R\left(G_{1}, G_{2}, \ldots, G_{t}\right)$ denotes the smallest integer $r$ with the property that whenever the edges of a complete graph on at least $r$ vertices are partitioned into $t$ colour classes, there is an integer $1 \leq i \leq t$ such that the $i$ th colour class contains a subgraph isomorphic to $G_{i}$. Such a subgraph will be referred to as a monochromatic subgraph in the $i$ th colour.

\footnotetext{
${ }^{1}$ Research partially supported by Hungarian Scientific Research Grant OTKA T043631.
} 
In the special case, when each $G_{i}=K_{k_{i}}$ is a complete graph on $k_{i}$ vertices, we will simply write $R\left(k_{1}, k_{2}, \ldots, k_{t}\right)$ for $R\left(G_{1}, G_{2}, \ldots, G_{t}\right)$. In general, if $G_{i}$ has $k_{i}$ vertices, then the existence of $R\left(G_{1}, G_{2}, \ldots, G_{t}\right)$ follows directly from that of $R\left(k_{1}, k_{2}, \ldots, k_{t}\right)$, the latter was first observed and applied to formal logic by Ramsey [13]. For more on Ramsey theory in general, we refer to the monograph $[6]$ and the collection of survey articles $[12,14]$.

A geometric graph is a graph drawn in the plane so that every vertex corresponds to a point, and every edge is a closed straight-line segment connecting two vertices but not passing through a third. The $\left(\begin{array}{l}n \\ 2\end{array}\right)$ segments determined by $n$ points in the plane, no three of which are collinear, form a complete geometric graph with $n$ vertices. A geometric graph is convex if its vertices correspond to those of a convex polygon. Further, we say that a subgraph of a geometric graph is non-crossing, if no two of its edges have an interior point in common.

For a sequence of graphs $G_{1}, G_{2}, \ldots, G_{t}$, the geometric Ramsey number that we denote by $R_{g}\left(G_{1}, G_{2}, \ldots, G_{t}\right)$ is defined as the smallest integer $r$ with the property that whenever the edges of a complete geometric graph on at least $r$ vertices are partitioned into $t$ colour classes, the $i$ th colour class contains a noncrossing copy of $G_{i}$, for some $1 \leq i \leq t$. The number $R_{c}\left(G_{1}, G_{2}, \ldots, G_{t}\right)$ denotes the corresponding number if we restrict our attention to convex geometric graphs only. These concepts have been introduced by Károlyi, Pach and Tóth in [9] and further explored in [10] and [11].

These numbers exist if and only if each graph $G_{i}$ is outerplanar, that is, can be obtained as a subgraph of a triangulated cycle (convex $n$-gon triangulated by non-crossing diagonals). The necessity of the condition is obvious, whereas the 'if part' is implied by the following theorem, based on a result of Gritzmann et al. [7].

Theorem 1. Let, for each $1 \leq i \leq t, G_{i}$ denote an outerplanar graph on $k_{i}$ vertices. Then

$$
R\left(G_{1}, \ldots, G_{t}\right) \leq R_{c}\left(G_{1}, \ldots, G_{t}\right) \leq R_{g}\left(G_{1}, \ldots, G_{t}\right) \leq R\left(k_{1}, \ldots, k_{t}\right) .
$$

Proof. Only the last inequality needs verification. For that end, consider any complete geometric graph $G$ on $R\left(k_{1}, k_{2}, \ldots, k_{t}\right)$ vertices whose edges are coloured using $t$ different colours. Then there is an integer $1 \leq i \leq t$ such that $G$ contains a 
monochromatic complete geometric graph $H_{i}$ on $k_{i}$ vertices in the $i$ th colour. The fact that $H_{i}$ contains a noncrossing subgraph isomorphic to $G_{i}$ follows directly from the following remarkable property of outerplanar graphs:

Theorem 2 ([7]). Let $P$ be an arbitrary set of $n$ points in the plane in general position. For any outerplanar graph $H$ on $n$ vertices, there is a straight-line embedding $f$ of $H$ into the plane such that the vertex set of $f(H)$ is $P$ and no two edges of $f(H)$ cross each other.

Throughout this paper we use the following notation. We denote by $C_{k}$ a cycle of $k$ vertices, $D_{k}$ a cycle of $k$ vertices triangulated from a vertex, $P_{k}$ a path of $k$ vertices, and $S_{k}$ a star of $k$ vertices. In addition, $M_{2 k}=k P_{2}$ will stand for any perfect matching on $2 k$ vertices. Results on geometric Ramsey numbers for paths and cycles were found by Károlyi et al. [10] and were extended in [11]. For example, if $k \geq 3$, then

$$
2 k-3=R_{c}\left(P_{k}, P_{k}\right) \leq R_{g}\left(P_{k}, P_{k}\right)=O\left(k^{3 / 2}\right) .
$$

Moreover, if $k$ and $\ell$ are integers larger than 2 , then

$$
\begin{aligned}
(k-1)(\ell-1)+1 & =R_{c}\left(C_{k}, P_{\ell}\right)=R_{g}\left(C_{k}, P_{\ell}\right) \leq R_{g}\left(C_{k}, C_{\ell}\right) \\
& \leq(k-1)(\ell-2)+(k-2)(\ell-1)+2 .
\end{aligned}
$$

For points in convex position, a similar result was obtained independently by Harborth and Lefmann [8]. We will consider a few ramifications in the following section, which in turn complete the solution of a problem studied in [2]. In Section 3 we prove that in the case $k=3$ the upper bound in the above estimate is sharp:

Theorem 3. For any integer $\ell \geq 3$,

$$
R_{c}\left(C_{3}, C_{\ell}\right)=R_{g}\left(C_{3}, C_{\ell}\right)=R_{g}\left(D_{3}, D_{\ell}\right)=3 \ell-3 .
$$

Note that the only further example when the exact value of either $R_{c}\left(C_{k}, C_{\ell}\right)$ or $R_{g}\left(C_{k}, C_{\ell}\right)$ is known is in the case $k=\ell=4$, namely $R_{c}\left(C_{4}, C_{4}\right)=R_{g}\left(C_{4}, C_{4}\right)=$ 14 , see $[2,10]$. It would be very interesting to see if the upper estimate in the above inequality is tight in general. 
Finally we study the existence of large monochromatic matchings in multicoloured complete graphs. It is proved in [4] that

$$
R\left(M_{2 k_{1}}, M_{2 k_{2}}, \ldots, M_{2 k_{t}}\right)=\sum_{i=1}^{t} k_{i}+\max _{1 \leq i \leq t} k_{i}-t+1 .
$$

This result, in the case $t=2$ has been extended to geometric graphs as follows:

Theorem $4([9])$.

$$
R_{c}\left(M_{2 k}, M_{2 \ell}\right)=R_{g}\left(M_{2 k}, M_{2 \ell}\right)=R\left(M_{2 k}, M_{2 \ell}\right)=k+\ell+\max \{k, \ell\}-1 .
$$

It implies the following general upper bound for

$$
R_{g}^{(t)}\left(M_{2 k}\right)=R_{g}(\underbrace{M_{2 k}, \ldots, M_{2 k}}_{t \text { times }}) .
$$

\section{Theorem 5.}

$$
R_{g}^{(t)}\left(M_{2 k}\right) \leq\left\{\begin{array}{cl}
\frac{3 t}{2} k-\frac{3 t}{2}+2 & \text { for } t \text { even }, \\
\frac{3 t+1}{2} k-\frac{3 t+1}{2}+2 & \text { for } t \text { odd }
\end{array}\right.
$$

Proof. The result is immediate for $t=1$, and is true also for $t=2$ in view of Theorem 4. Assume thus first that $t=2 s \geq 4$ is even. Let $G$ be any $t$-coloured complete geometric graph on $N \geq \frac{3 t}{2} k-\frac{3 t}{2}+2$ vertices. Recolouring the edges of $G$ such that the edges coloured using any of the first $s$ colours are now coloured with a first new colour and the remaining edges with another new colour, we find that one of the new colour classes contains $s(k-1)+1$ pairwise disjoint edges, due to the fact that

$$
N \geq 3 s k-3 s+2=3(s(k-1)+1)-1=R_{g}\left(M_{2(s(k-1)+1)}, M_{2(s(k-1)+1)}\right) .
$$

It follows that one of the $s$ original colour classes that combine to this new colour class contains $k$ pairwise disjoint edges. Similarly, for $t=2 s+1 \geq 3$, we have

$$
R_{g}^{(t)}\left(M_{2 k}\right) \leq R_{g}\left(M_{2(s(k-1)+1)}, M_{2((s+1)(k-1)+1)}\right)=\frac{3 t+1}{2} k-\frac{3 t+1}{2}+2 .
$$

In particular, $R_{g}\left(M_{2 k}, M_{2 k}, M_{2 k}\right) \leq 5 k-3$ and $R_{g}\left(M_{2 k}, M_{2 k}, M_{2 k}, M_{2 k}\right) \leq 6 k-4$. In Section 4 we will prove that the latter bound is sharp: 
Theorem 6. For every positive integer $k$ we have

$$
R_{c}\left(M_{2 k}, M_{2 k}, M_{2 k}, M_{2 k}\right)=R_{g}\left(M_{2 k}, M_{2 k}, M_{2 k}, M_{2 k}\right)=6 k-4 .
$$

Combining this result with the obvious inequality

$$
R_{c}^{(t+1)}\left(M_{2 k}\right) \geq R_{c}^{(t)}\left(M_{2 k}\right)+(k-1)
$$

we obtain the following general lower bound:

Theorem 7. If $t \geq 4$ and $k$ are positive integers, then $R_{c}^{(t)}\left(M_{2 k}\right) \geq(t+2) k-t$.

We give the following essential improvement upon this result.

Theorem 8. For arbitrary integers $t \geq 2$ and $k \geq 6 t-10$ we have

$$
R_{c}^{(t)}\left(M_{2 k}\right)>\frac{6}{5} t k
$$

In [1], Araujo et al. studied the chromatic number of some geometric Kneser graphs. For example, given $n$ points in convex position in the plane, let $G_{n}$ be the graph whose vertices are the $\left(\begin{array}{l}n \\ 2\end{array}\right)$ line segments determined by the points, two such vertices connected by an edge in $G_{n}$ if and only if the corresponding line segments are disjoint.

Theorem 9 ([1]).

$$
2\left\lfloor\frac{n+1}{3}\right\rfloor-1 \leq \chi\left(G_{n}\right) \leq \min \left\{n-2, n-\frac{\log n}{2}\right\} .
$$

In this result, the lower bound is derived as a consequence of Theorem 4. Note that any improvement upon the upper bound in Theorem 5 , even when $R_{g}$ is replaced by $R_{c}$, would have an impact on the lower bound in the above theorem. However, Theorem 8 shows that no improvement beyond $\frac{5}{6} n$ could be obtained this way. 


\section{General Estimates}

In this section we apply the methods of $[10,11]$ to obtain a few general estimates concerning asymmetric geometric graph Ramsey numbers.

Theorem 10. If $k, \ell \geq 2$ and $H$ is an outerplanar graph with $\ell$ vertices, then

(i) $R_{g}\left(S_{k}, H\right) \leq(k-1)(\ell-1)+1$ and

(ii) $R_{g}\left(P_{k}, H\right) \leq(k-1)(\ell-1)+1$.

Proof. The proof of the first inequality depends on a result of Chvátal [3] claiming that $R\left(T_{k}, K_{\ell}\right)=(k-1)(\ell-1)+1$ for every fixed tree $T_{k}$ on $k$ vertices. Coupled with Theorem 2 it implies that any complete geometric graph with at least $(k-$ $1)(\ell-1)+1$ vertices whose edges are coloured with red and blue contains either a blue noncrossing copy of $H$ or a red copy of $S_{k}$ whose edges cannot cross anyway.

To prove the second statement, consider a complete geometric graph on at least $(k-1)(\ell-1)+1$ vertices whose edges are coloured with red and blue. Let $p_{1}, p_{2}, \ldots, p_{(k-1)(\ell-1)+1}$ be vertices of this graph, listed in increasing order of their $x$-coordinates, which we may assume to be all distinct. A path $p_{i_{1}} p_{i_{2}} \ldots p_{i_{j}}$ is said to be monotone if $i_{1}<i_{2}<\ldots<i_{j}$. Introduce a partial ordering on these vertices as follows. Let $p_{i}<p_{j}$ if and only if $i<j$, and there is a monotone red path connecting $p_{i}$ to $p_{j}$. By Dilworth's theorem [5], one can find either $k$ elements that form a totally ordered subset $Q \subseteq\left\{p_{1}, p_{2}, \ldots, p_{(k-1)(\ell-1)+1}\right\}$, or $\ell$ elements that are pairwise incomparable. In the first case there is a monotone red path visiting every vertex of $Q$, it contains a red subpath on $k$ vertices whose edges do not cross. Note that any two incomparable elements are connected by a blue edge. Thus one finds a complete blue subgraph on $\ell$ vertices in the second case. According to Theorem 2 it contains a noncrossing copy of $H$.

Theorem 11. Let $G$ be a connected outerplanar graph with $k \geq 2$ vertices and $H$ any outerplanar graph that contains a cycle of length $\ell$. Then

$$
(k-1)(\ell-1)+1 \leq R_{c}(G, H) .
$$

Proof. Take $(k-1)(\ell-1)$ points on a circle and partition them into $\ell-1$ groups, each containing $k-1$ consecutive points. Colour with blue all edges between points in different groups, and colour with red all edges between points belonging to the same group. It follows that the two-coloured geometric graph thus obtained 
does not contain a noncrossing blue copy of $H$. Indeed, any noncrossing blue cycle contains at most one point from each group, hence it can not have more than $\ell-1$ points. On the other hand, all vertices of a red connected subgraph must be from the same group, so there is no such graph with more than $k-1$ points.

Putting the lower and upper bounds together we obtain the following result.

Corollary 12. Let $k \geq 2$ be an integer and $H$ any outerplanar graph on $\ell$ vertices that contains a cycle of length $\ell$. Then

$$
R_{c}\left(S_{k}, H\right)=R_{c}\left(P_{k}, H\right)=R_{g}\left(S_{k}, H\right)=R_{g}\left(P_{k}, H\right)=(k-1)(\ell-1)+1 .
$$

In fact, the following general statement may be true.

Conjecture 13. Let $T_{k}$ denote a tree of $k \geq 2$ vertices and $H$ any outerplanar graph on $\ell$ vertices that contains a cycle of length $\ell$. Then

$$
R_{c}\left(T_{k}, H\right)=R_{g}\left(T_{k}, H\right)=(k-1)(l-1)+1 .
$$

The lower bound follows directly from Theorem 11.

Finally, let $G$ and $H$ denote arbitrary outerplanar graphs on $k$ and $\ell$ vertices, respectively. According to Theorem 1,

$$
R_{g}(G, H) \leq R(k, \ell) \leq\left(\begin{array}{c}
k+\ell-2 \\
\ell-1
\end{array}\right) .
$$

We do not know, if this upper bound can be essentially reduced in general. It is the case, however, if each graph is part of a cycle triangulated from one vertex.

Theorem 14. For arbitrary integers $k, \ell \geq 3$ we have

$$
R_{g}\left(D_{k}, D_{\ell}\right) \leq(k-2)(\ell-1)+(k-1)(\ell-2)+2 .
$$

Proof. Let $P$ denote the vertex set of a complete geometric graph of at least $(k-2)(\ell-1)+(k-1)(\ell-2)+2$ vertices, whose edges are coloured with red and blue. Let $p$ be a vertex of the convex hull of $P$. Consider the edges incident to $p$, either at least $(k-2)(\ell-1)+1$ of them are red, or at least $(k-1)(\ell-2)+1$ of them are blue. Suppose, without any loss of generality, that the first possibility is the case. Let $p_{1}, p_{2}, \ldots, p_{(k-2)(\ell-1)+1}$ be vertices of $P$, listed in clockwise order of visibility from $p$, such that each edge $p p_{i}$ is red. We say that a path $p_{i_{1}} p_{i_{2}} \ldots p_{i_{j}}$ is monotone if $i_{1}<i_{2}<\ldots<i_{j}$. Define a partial ordering on the vertices 
$p_{1}, p_{2}, \ldots, p_{(k-2)(\ell-1)+1}$ as follows. Let $p_{i}<p_{j}$ if and only if $i<j$, and there is a monotone red path connecting $p_{i}$ to $p_{j}$. Applying Dilworth's theorem again, there are either $k-1$ elements that form a linearly ordered subset, or $\ell$ elements that are pairwise incomparable. In the first case there is a monotone red path $q_{1} q_{2} \ldots q_{k-1}$, and we can complete it to a noncrossing red cycle $p q_{1} q_{2} \ldots q_{k-1} p$ of length $k$, triangulated from the point $p$. In the second case there is a complete blue subgraph on $\ell$ vertices, and it follows from Theorem 2 that it contains a blue noncrossing copy of $D_{\ell}$.

For any convex drawing $D$ of a given graph, let $r_{c}(D)$ denote the smallest integer $r$ such that every two-colouring of the diagonals and sides of a convex $r$-gon contains a monochromatic copy of $D$. In [2], Bialostocki and Harborth determined $r_{c}(D)$ for all convex drawings $D$ of graphs with 4 vertices, except one. What they proved about that exceptional case can be rephrased as $14 \leq$ $R_{c}\left(D_{4}, D_{4}\right) \leq 16$. From Theorem 14 it follows that

$$
R_{c}\left(D_{4}, D_{4}\right)=R_{g}\left(D_{4}, D_{4}\right)=14,
$$

thus completing the investigation initiated in [2].

\section{Noncrossing Cycles Versus Triangles}

Proof of Theorem 3. It follows from Theorem 14 that $R_{c}\left(C_{3}, C_{\ell}\right) \leq R_{g}\left(C_{3}, C_{\ell}\right) \leq$ $R_{g}\left(D_{3}, D_{\ell}\right) \leq 3 \ell-3$. In order to prove that $R_{c}\left(C_{3}, C_{\ell}\right) \geq 3 \ell-3$, we construct a two-coloured geometric graph with $3 \ell-4$ vertices in convex position which does not have a monochromatic red triangle, nor a blue noncrossing cycle of $\ell$ vertices. According to the parity of $\ell$, we distinguish between two cases.

Assume first that $\ell$ is odd. Put $N=3 \ell-4$ and let $x_{1}, x_{2}, \ldots, x_{3 \ell-4}$ be the vertices of a convex $N$-gon $P$, listed in clockwise order. For an integer $n$, let $n^{*}$ denote the integer between 0 and $N-1$ obtained reducing $n$ modulo $N$. Colour the edge $x_{i} x_{j}$ red if $\min \left\{(j-i)^{*},(i-j)^{*}\right\}=2 s-1$ for some integer $s$ that satisfies $1 \leq 2 s-1 \leq \ell-2$, and colour it blue otherwise. This way we obtain a well defined two-colouring of the complete convex geometric graph $G$ whose vertices are $x_{1}, x_{2}, \ldots, x_{3 \ell-4}$. If $x_{i}$ and $x_{j}$ are consecutive vertices in the clockwise order of some convex polygon $Q$ whose vertices are among the vertices of $P$, then we say that the (combinatorial) length of the side $x_{i} x_{j}$ is $(j-i)^{*}$. 
We say that a side is short if its length does not exceed $\ell-2$, it is called long otherwise. Note that all short red edges are of odd length and all short blue edges are of even length.

Suppose that $x_{i}, x_{j}, x_{k}$ are the vertices of a triangle in the clockwise order. Since $3(\ell-2)<3 \ell-4$, it cannot be that all edges are short. If $x_{k} x_{i}$ is a long red edge, then $(k-i)^{*}$ is an odd integer not exceeding $\ell-2$. Then both edges $x_{i} x_{j}$ and $x_{j} x_{k}$ must be short and one of them has an even length, which implies it cannot be red. Consequently, $G$ does not contain a red triangle.

Next let $x_{i_{1}} x_{i_{2}}, \ldots, x_{i_{\ell}}$ be the vertices of a noncrossing cycle of length $\ell$. If it has a long edge, then the sum of the lengths of the remaining edges is at most $(3 \ell-4)-(\ell-1)=2 \ell-3$, thus one of the remaining edges is of length 1 , hence cannot be blue. On the other hand, if all edges are short, then since the sum of their lengths, $3 \ell-4$ is an odd number, one of them must have an odd length and thus cannot be blue. All in all, $G$ contains no non-crossing blue $C_{\ell}$ either.

The case when $\ell$ is even is slightly more complicated. First we construct a two-colouring of a complete convex geometric graph $G^{\prime}$ on $N=3 \ell-3$ vertices, obtained by inserting an auxiliary vertex $x_{0}$ between $x_{3 \ell-4}$ and $x_{1}$. Using the terminology of the previous case, we colour all short edges of odd length red. This time we will call medium edges those whose length is $\ell-1$, and the following medium edges we also color red: $x_{2 i+1} x_{2 i+\ell}$ for $0 \leq i \leq \ell-2$ and $x_{i^{*}} x_{(i+\ell-1)^{*}}$ for $2 \ell-2 \leq i \leq 3 \ell-3$. The other edges are coloured blue. Note that medium edges also have an odd length. This time an edge is called long if its length is at least $\ell$.

Assume that $G^{\prime}$ contains a red triangle $\Delta$. The same argument we had in the previous case confirms that it cannot have a long edge. Thus all edges are of length at most $\ell-1$ and these 3 lengths add up to $N=3 \ell-3$. Thus all edges of $\Delta$ are medium. $\Delta$ then must have an edge $x_{i} x_{i+\ell-1}$ for some $1 \leq i \leq \ell-1$ in which case $i$ is odd and $i+\ell-1 \leq 2 \ell-2$ is even. If $i+\ell-1<2 \ell-2$, then the edge $x_{i+\ell-1} x_{i+2 \ell-2}$ is not red. It follows that the vertices of $\Delta$ are $x_{\ell-1}, x_{2 \ell-2}$ and $x_{0}$.

Suppose now that $C$ is a noncrossing blue cycle of length $\ell$ in $G^{\prime}$. As in the case when $\ell$ was odd, we can argue that $C$ cannot have only short edges, by parity reason. Also, every short edge has a length at least 2 , thus if it has a non-short edge, then an elementary calculation implies that $C$ has exactly one medium edge 
and $\ell-1$ other edges, each of length 2 . It follows from the construction that the endpoints of the blue medium edge are $x_{2 i}$ and $x_{2 i+\ell-1}$ for some $1 \leq i \leq \ell-2$. Accordingly, one vertex of $C$ must be $x_{0}$.

Finally, let $G$ be the two-coloured convex geometric graph induced by $G^{\prime}$ on the vertex set $x_{1}, x_{2}, \ldots, x_{3 \ell-4}$. Were $\Delta$ a red triangle in $G$, it also would be a red triangle in $G^{\prime}$, thus it would necessarily contain the vertex $x_{0}$, a contradiction. A similar argument shows that $G$ does not contain a noncrossing blue $C_{\ell}$ either.

\section{Noncrossing Matchings in 4-Coloured Geometric Graphs}

Proof of Theorem 6. In view of Theorems 1 and 5, we only have to present a fourcolouring of the edges of a complete convex geometric graph on $6 k-5$ vertices such that there are no $k$ (geometrically) disjoint edges of the same colour. Let the vertices of the graph be points on a circle, listed in clockwise order as

$$
b_{1}, \ldots, b_{k-1}, r_{1}, r_{1}^{\prime}, \ldots, r_{k-1}, r_{k-1}^{\prime}, g_{1}, \ldots, g_{k-1}, p_{k}, p_{k-1}^{\prime}, p_{k-1}, \ldots, p_{1}^{\prime}, p_{1} .
$$

For simplicity, call the first $k-1$ vertices blue, the next $2 k-2$ vertices red, the $k-1$ vertices that follow green, and the last $2 k-1$ vertices purple. We colour the edges of the corresponding complete convex geometric graph using the same set of colours as follows.

First, use red to colour each edge connecting two red vertices, and in addition for each edge that connects a red vertex $r_{i}^{\prime}$ to any other vertex. Similarly, every edge between two purple points are coloured purple as well as any edge starting at some vertex $p_{i}^{\prime}(1 \leq i \leq k-1)$. Edges of the form $r_{i}^{\prime} p_{j}^{\prime}$ may be coloured either purple or red. Next, assign blue to each yet uncoloured edge starting at a blue vertex, and similarly, use the green colour for each edge connecting some green vertex to any other vertex except the vertices $r_{i}^{\prime}, p_{i}^{\prime}$. Again, edges of the form $b_{i} g_{j}$ may be either blue or green. Finally, we colour the edge $p_{i} r_{j}$ blue if and only if $i+j \leq k$, else we colour it green. This way we have assigned a colour to each edge of the complete graph.

This graph does not contain $k$ pairwise disjoint purple edges, as it follows directly from the following lemma.

Lemma 15. Assume that $P=p_{1} p_{1}^{\prime} p_{2} p_{2}^{\prime} \ldots p_{n}$ is a convex polygon, and $Q=$ $\left\{q_{1}, q_{2}, \ldots, q_{m}\right\}$ is a set of points lying in the interior of the convex (finite or 
infinite) domain bounded by the line segment $p_{1} p_{n}$, the extension of the line segment $p_{1} p_{1}^{\prime}$ beyond the point $p_{1}$, and the extension of the line segment $p_{n-1}^{\prime} p_{n}$ beyond $p_{n}$. Consider all the line segments that are either connecting two vertices of $P$, or connect some point $p_{i}^{\prime}$ to a point $q_{j}$. Then no $n$ of these line segments can be pairwise disjoint.

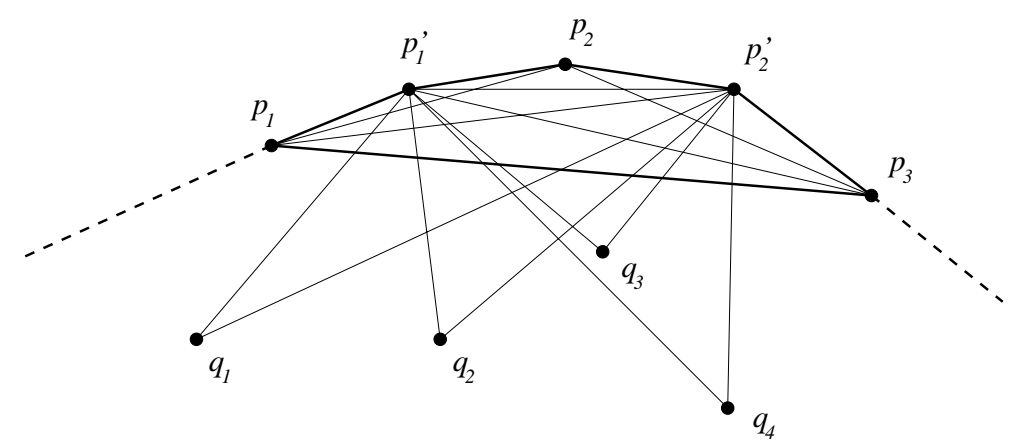

Figure 1

Proof. We proceed by induction on $n$. The claim being obvious for $n=2$, we assume that $n>2$, and that we have already proved the statement for smaller values of $n$. Consider a set $S$ of $\ell$ pairwise disjoint segments. If each of them has an endpoint in the set $\left\{p_{1}^{\prime}, p_{2}^{\prime}, \ldots, p_{n-1}^{\prime}\right\}$, then clearly $\ell \leq n-1$. Otherwise one of the segments is of the form $s=p_{i} p_{j}$ with $1 \leq i<j \leq n$. Let $S_{0}$ be the set of all line segments in $S$ whose both endpoints belong to the set $\left\{p_{i}, p_{i}^{\prime}, \ldots, p_{j}\right\}$. Obviously, $s \in S_{0}$, and $\left|S_{0}\right| \leq j-i$. If $i=1$ and $j=n$, then clearly $S=S_{0}$, and we are done. If $i>1$ but $j=n$, then $S^{\prime}=S \backslash S_{0}$ is a set of pairwise disjoint line segments in the configuration determined by the vertices of $P^{\prime}=p_{1} p_{1}^{\prime} p_{2} p_{2}^{\prime} \ldots p_{i}$ and the points of $Q^{\prime}=Q$, just as in the lemma. Since certainly $2 \leq i \leq n-1$ in this case, it follows from the induction hypothesis that $\ell-\left|S_{0}\right|=\left|S^{\prime}\right|<i$, that is, $\ell<i+\left|S_{0}\right| \leq n$. We can argue similarly if $i=1$ and $j<n$. Finally, if $1<i<j<n$, then we may consider $P^{\prime}=p_{1} p_{1}^{\prime} \ldots p_{i} p_{j}^{\prime} p_{j+1} p_{j+1}^{\prime} \ldots p_{n}, Q^{\prime}=Q$ and $S^{\prime}=S \backslash S_{0}$ to find, based on the induction hypothesis, that $\left|S^{\prime}\right|<n-(j-i)$. It follows, that

$$
|S|=\left|S^{\prime}\right|+\left|S_{0}\right|<(n-j+i)+(j-i)=n,
$$

completing the induction step. 
Similarly, the graph does not contain a noncrossing red $M_{2 k}$ either.

Assume next that the graph contains $n$ pairwise disjoint blue edges, and consider only these edges. If they all have a blue endpoint, then clearly $n \leq k-1$. Otherwise we assume that there are $\ell \geq 1$ edges of the form $p_{i} r_{j}$. Since they do not cross, we can list them as $p_{i_{1}} r_{j_{1}}, \ldots, p_{i_{\ell}} r_{j_{\ell}}$ so that $i_{1}<\ldots<i_{\ell}$ and $j_{1}<\ldots<j_{\ell}$. It follows that

$$
i_{1}+j_{1}+2(\ell-1) \leq i_{\ell}+j_{\ell} \leq k .
$$

The endpoints of the other $n-\ell$ edges must lie in the vertex set

$$
\left\{b_{1}, b_{2}, \ldots, b_{k-1}, p_{1}, p_{2}, \ldots, p_{i_{1}-1}, r_{1}, r_{2}, \ldots, r_{j_{1}-1}\right\} .
$$

Consequently,

$2(n-\ell) \leq(k-1)+\left(i_{1}-1\right)+\left(j_{1}-1\right) \leq(k-1)+k-2(\ell-1)-2=2(k-\ell)-1$.

It follows that $n<k$ holds in this case, too.

Due to the symmetry in the construction, the existence of a noncrossing green matching on $2 k$ vertices can be excluded by a similar argument.

The proof of Theorem 8 depends on the following lemma that we found in the pursuit of a possible generalization of the previous arguments.

Lemma 16. Let $r$ denote any positive integer. If $k \geq 4 r$, then the edges of a complete convex geometric graph on $n=(6 r+6) k-(12 r+12)$ vertices can be coloured with $t=5 r+4$ colours so that it does not contain a monochromatic crossing-free matching of size $k$.

Indeed, Theorem 4 and the inequality (1) implies

$$
R_{c}^{(2)}\left(M_{2 k}\right)=3 k-1>\frac{12}{5} k
$$

for $k \geq 2$ and

$$
R_{c}^{(3)}\left(M_{2 k}\right) \geq 4 k-2>\frac{18}{5} k
$$

for $k \geq 8$ (in fact, even for $k \geq 6$ ). For the values $4 \leq t \leq 8$, the statement of Theorem 8 follows directly from Theorem 7 . Finally if $t=5 r+4+i$ for some integers $r \geq 1$ and $0 \leq i \leq 4$, then Lemma 16 coupled with relation (1) implies

$$
R_{c}^{(t)}\left(M_{2 k}\right) \geq(6 r+6+i) k-(12 r+11+i)>\frac{6}{5} k t
$$

for any $k \geq 6 t-10$. It only remains to prove the lemma. 
Proof of Lemma 16. Choose $n$ points on a circle and partition them into $4 r+4$ sets of consecutive points

$$
B_{i}=\left\{b_{i 1}, b_{i 2}, \ldots, b_{i, k-1}\right\}, \quad R_{i}=\left\{r_{i 1}, r_{i 1}^{\prime}, r_{i 2}, r_{i 2}^{\prime}, \ldots, r_{i, k-2}\right\}
$$

for $1 \leq i \leq 2 r+2$. We choose each numbering in clockwise direction. Thus $\left|B_{i}\right|=k-1$ and $\left|R_{i}\right|=2 k-5$. There will be $4 r+4$ corresponding colours that we denote by $\mathcal{B}_{i}$ and $\mathcal{R}_{i}$, respectively.

First, use the colour $\mathcal{R}_{i}$ for any edge connecting two vertices in $R_{i}$, and also for any edge starting at some vertex $r_{i j}^{\prime}$, where $1 \leq j \leq k-3$. Edges of the form $r_{i j}^{\prime} r_{u v}^{\prime}$ may belong to either $\mathcal{R}_{i}$ or $\mathcal{R}_{u}$. It follows from Lemma 15 that the colour class $\mathcal{R}_{i}$ contains at most $k-3$ pairwise disjoint edges.

Next, we use the colour $\mathcal{B}_{i}$ to colour each, so far uncoloured edge that has a vertex in $B_{i}$. Edges of the form $b_{i j} b_{u v}$ may belong to either $\mathcal{B}_{i}$ or $\mathcal{B}_{u}$. In addition, we assign the colour $B_{i}$ to each edge of the form $r_{i^{*} j} r_{i j^{\prime}}$ whenever $j^{\prime} \leq j$ and the number $1 \leq i^{*} \leq 2 r+2$ satisfies $i^{*}+1 \equiv i(\bmod 2 r+2)$. As in the proof of Theorem 6 , it follows that the colour class $B_{i}$ cannot contain $k$ pairwise disjoint edges either.

Finally, we use $r$ further colours $\mathcal{C}_{1}, \ldots, \mathcal{C}_{r}$ to colour the remaining edges, all of which are of the form $r_{i j} r_{u v}$ for some $i \neq u$. We have $(2 r+2)(k-2)$ points

$$
r_{11}, r_{12}, \ldots, r_{1, k-2}, r_{21}, \ldots, r_{2, k-2}, r_{2 r+2,1}, \ldots, r_{2 r+2, k-2}
$$

positioned along a circle $K$. Without any loss of generality, we may assume that these are the vertices of a regular polygon $x_{1} x_{2} \ldots x_{(2 r+2)(k-2)}$ whose center we denote by $O$. For $2 \leq \ell \leq(r+1)(k-2)$, we say that the diagonal $x_{i} x_{j}$ is of length $\ell$ if $j-i \equiv \ell(\bmod (2 r+2)(k-2))$. In this case we write $\ell=l\left(x_{i} x_{j}\right)$. We only have to colour those diagonals whose length is at least $k-1$.

This is done as follows. For $k-1 \leq \ell \leq(r+1)(k-2)$ and $1 \leq i \leq r$, let a diagonal of length $\ell$ belong to $\mathcal{C}_{i}$ if and only if $\ell \equiv 2 i-1(\bmod 2 r)$ or $\ell \equiv 2 i$ $(\bmod 2 r)$. To complete the proof of Lemma 16 we only have to show that $\mathcal{C}_{i}$ does not contain $k$ pairwise disjoint diagonals for $1 \leq i \leq r$.

Thus let $1 \leq i \leq r$, and consider a set $\mathcal{M}$ of pairwise disjoint diagonals in $\mathcal{C}_{i}$. The diagonal $e \in \mathcal{M}$ is called extremal if $\mathcal{M}$ lies in a closed half plane supported by $e$. Denote by $q$ the number of such extremal edges. We may suppose that $|\mathcal{M}|>1$, and thus $q \geq 2$. For any line $L$ through $O$, fix a pair of disjoint half planes $H_{L}^{+}$and $H_{L}^{-}$supported by $L$ so that the first one is closed and the other 
is open, and introduce

$$
\mathcal{H}=\left\{H_{L}^{+}, H_{L}^{-} \mid L \text { is a line through } O\right\}
$$

For edges $e, f \in \mathcal{M}$, denote by $K_{\text {ef }}$ the closed region that $e$ and $f$ cut out of the circle $K$, it degenerates to a segment when $e=f$. We say that $e$ and $f$ are neighbours, if $K_{\text {ef }}$ does not contain any member of $\mathcal{M}$ except $e$ and $f$. A sequence $e_{1}, e_{2}, \ldots, e_{u}$ of pairwise different edges in $\mathcal{M}$ forms a contiguous set if they all belong to the same half plane $H$ for some $H \in \mathcal{H}$, and $e_{j}$ and $e_{j+1}$ are neighbours for every $1 \leq j \leq u-1$. There is a unique partition of $\mathcal{M}$ into maximal contiguous sets $\mathcal{M}_{1}, \ldots, \mathcal{M}_{m}$.

Claim 17. $m \leq 2 q-2$.

Proof. We say that set $\mathcal{M}_{j}$ is extremal, if it contains an extremal edge. Since $q \geq 2$, we are done if $m \leq 2$. On the other hand, if $m \geq 2$, then each extremal set has exactly one extremal edge. For each $1 \leq j \leq m$ there is a unique pair of edges $e_{j}, f_{j} \in \mathcal{M}_{j}$ such that $\mathcal{M}_{j}$ is contained in $K_{j}=K_{e_{j} f_{j}}$. If $e_{j} \neq f_{j}$, the shorter of the two diagonals will be denoted by $e_{j}$, its midpoint by $E_{j}$.

We build up a rooted tree $T$ as follows. The root of the tree is the point $O$, its further vertices are $E_{1}, \ldots, E_{m}$. If by any chance $O=E_{j}$ for some $j$ (which only can happen if $\left|\mathcal{M}_{j}\right|=1$ ), then we extend $e_{j}$ to a line $L$ and slightly move $O$ around so that it no longer lies in $H_{L}^{+}$.

First, we connect $O$ to $E_{j}$ if and only if the segment $O E_{j}$ only intersects such diagonals in $\mathcal{M}$ that belong to $\mathcal{M}_{j}$. This way we get the level one vertices of $T$. For any $E_{u}$ not already in $T$ there is a (unique) vertex $E_{j}$ on level one such that $e_{j}$ separates $\mathcal{M}_{u}$ from $O$. For each level one vertex $E_{j}$ such that $\mathcal{M}_{j}$ is not extremal there are at least two different vertices $E_{u}$ not yet in $T$ such that the segment $E_{j} E_{u}$ only intersects such diagonals in $\mathcal{M}$ that belong to $\mathcal{M}_{u}$. Connecting each such vertex $E_{u}$ with $E_{j}$ we arrive at the level two vertices of $T$. Iterating this process we arrive at the tree $T$ rooted at $O$ that has $m+1$ vertices, $E_{j}$ is a leaf if and only if $e_{j}$ is an extremal edge, and any vertex $E_{j}$ which is not a leaf has degree at least 3 .

Assume first that the root $O$ has a degree at least 2. In this case $\mathcal{M}_{j}$ is extremal if and only if $e_{j}$ is extremal, and thus $T$ has exactly $q$ leaves and $m-q$ vertices different from $O$ such that each of them has a degree at least 3. Since $T$ 
has exactly $m$ edges, double counting yields

$$
2 m \geq q+2+3(m-q),
$$

and the result follows.

If $\operatorname{deg}(O)=1$, then let $E_{j}$ be the unique neighbour of $O$. In this case $\mathcal{M}_{j}$ is also extremal, its extremal edge being $f_{j}$. This time the number of leaves is $q-1$, thus $T$ has $q$ degree one vertices, and each further vertex of $T$ has a degree at least 3. It follows that

$$
2 m \geq q+3(m+1-q),
$$

that is, $m \leq 2 q-3$.

Write $s_{j}=\left|\mathcal{M}_{j}\right|$, and denote by $n_{j}$ the number of vertices $x_{u}$ that lie in $K_{j}$.

Claim 18. If $1 \leq j \leq m$, then $n_{j} \geq 2 r s_{j}-(2 r-1)$.

Proof. List the diagonals $d_{1}, \ldots, d_{s_{j}}$ in $M_{j}$ so that $l\left(d_{1}\right)<\ldots<l\left(d_{s_{j}}\right)$. Note that $l\left(d_{u+1}\right)>l\left(d_{u}\right)+1$ for any $1 \leq u<s_{j}$. For every $u$ there is an integer $k_{u}$ such that $l\left(d_{u}\right)=2 r k_{u}+m_{u}$, where $m_{u}$ is either $2 i-1$ or $2 i$. It follows that $k_{u+1}>k_{u}$ for every $1 \leq u<s_{j}$, thus $l\left(d_{s_{j}}\right)-l\left(d_{1}\right) \geq 2 r\left(s_{j}-1\right)-1$ and consequently $n_{j} \geq 2 r\left(s_{j}-1\right)+1$.

Since the regions $K_{1}, K_{2}, \ldots, K_{m}$ are pairwise disjoint, each vertex $x_{u}$ can belong to at most one of them. Moreover, each extremal edge cuts off at least $k-2$ consecutive vertices that cannot belong to any $K_{j}$. Consequently,

$$
(2 r+2)(k-2) \geq \sum_{j=1}^{m} n_{j}+q(k-2) \geq 2 r|\mathcal{M}|-m(2 r-1)+q(k-2),
$$

according to Claim 18 and the equality $|\mathcal{M}|=s_{1}+\ldots+s_{m}$. It follows that

$$
\begin{aligned}
2 r(k-|\mathcal{M}|) & \geq(q-2)(k-2)-m(2 r-1)+4 r \\
& =(q-2)(k-2)-(m-2)(2 r-1)+2 \\
& \geq(q-2)(k-2-2(2 r-1))+2 \\
& =(q-2)(k-4 r)+2 \\
& >0 .
\end{aligned}
$$

Thus $|\mathcal{M}|<k$, and the proof of Lemma 16 is complete. 


\section{REFERENCES}

[1] G. Araujo, A. Dumitrescu, F. Hurtado, M. Noy, and J. Urrutia, On the chromatic number of some geometric Kneser graphs, Comput. Geom. Th. Appl. 32 (2005) 59-69

[2] A. Bialostocki And H. Harborth, Ramsey colorings for diagonals of convex polygons, Abh. Braunschweig. Wiss. Ges. 47 (1996) 159-163

[3] V. ChVÁtal, Tree - complete graph Ramsey numbers, J. Graph Theory 1 (1977) 93

[4] E.J. Cockayne and P.J. Lorimer, The Ramsey number for stripes, J. Austral. Math. Soc. A 19 (1975) 252-256

[5] R.P. Dilworth, A decomposition theorem for partially ordered sets, Annals Math. 51 (1950) 161-166

[6] R.L. Graham, B.L. Rothschild, and J.H. Spencer, Ramsey Theory, 2nd ed., John Wiley, New York (1990)

[7] P. Gritzmann, B. Mohar, J. Pach, And R. Pollack, Embedding a planar triangulation with vertices at specified points (solution to problem E3341), Amer. Math. Monthly 98 (1991) 165-166

[8] H. Harborth and H. Lefmann, Coloring arcs of convex sets, Discrete Math. 220 (2000) 107-117

[9] Gy. Károlyi, J. Pach, And G. Tóth, Ramsey-type results for geometric graphs. I, Discrete Comput. Geom. 18 (1997) 247-255

[10] Gy. Károlyi, J. Pach, G. Tóth, and P. Valtr, Ramsey-type results for geometric graphs. II, Discrete Comput. Geom. 20 (1998) 375-388

[11] Gy. Károlyi and V. Rosta, Generalized and geometric Ramsey numbers, Theoretical Comput. Sci. 263 (2001) 87-98

[12] J. Nešetřll And V. RödL (Eds.), Mathematics of Ramsey Theory, Algorithms and Combinatorics 5, Springer (1990)

[13] F.P. Ramsey, On a problem of formal logic, Proc. London Math. Soc. 30 (1930) 264-286

[14] V. Rosta, Ramsey theory applications, Electron. J. Combin. 11 (2004) Dynamic Survey DS13, 43 pages (electronic)

E-mail address: karolyi@cs.elte.hu ' Gyula Károlyi',

E-mail address: rosta@renyi.hu 'Vera Rosta'" 